\title{
Sexual Dimorphism of Short-Wavelength Photoreceptors in the Small White Butterfly, Pieris rapae crucivora
}

\author{
Kentaro Arikawa, ${ }^{1}$ Motohiro Wakakuwa, ${ }^{1}$ Xudong Qiu, ${ }^{1}$ Masumi Kurasawa, ${ }^{1}$ and Doekele G. Stavenga ${ }^{2}$ \\ ${ }^{1}$ Graduate School of Integrated Science, Yokohama City University, Yokohama 236-0027, Japan, and 'Department of Neurobiophysics, University of \\ Groningen, NL-9747 AG Groningen, The Netherlands
}

The eyes of the female small white butterfly, Pieris rapae crucivora, are furnished with three classes of short-wavelength photoreceptors, with sensitivity peaks in the ultraviolet (UV) $\left(\lambda_{\max }=360 \mathrm{~nm}\right)$, violet $(\mathrm{V})\left(\lambda_{\max }=425 \mathrm{~nm}\right)$, and blue $(\mathrm{B})\left(\lambda_{\max }=453 \mathrm{~nm}\right)$ wavelength range. Analyzing the spectral origin of the photoreceptors, we isolated three novel mRNAs encoding opsins corresponding to shortwavelength-absorbing visual pigments. We localized the opsin mRNAs in the retinal tissue and found that each of the short-wavelengthsensitive photoreceptor classes exclusively expresses one of the opsin mRNAs. We, accordingly, termed the visual pigments $\operatorname{PrUV}$, PrV, and $\operatorname{PrB}$, respectively. The eyes of the male small white butterfly also use three classes of short-wavelength photoreceptors that equally uniquely express PrUV, PrV, and PrB. However, whereas the spectral sensitivities of the male photoreceptors with PrUV and PrB closely correspond to those of the female, the male photoreceptor expressing $\operatorname{PrV}$ has a double-peaked blue (dB) spectral sensitivity, strongly deviating from the spectral sensitivity of the female $\mathrm{V}$ photoreceptor. The male eyes contain a pigment that distinctly fluoresces under blue-violet as well as UV excitation light. It coexists with the $\mathrm{dB}$ photoreceptors and presumably acts as a spectral filter with an absorbance spectrum peaking at $416 \mathrm{~nm}$. The narrow-band spectral sensitivity of the male $\mathrm{dB}$ photoreceptors probably evolved to improve the discrimination of the different wing colors of male and female $P$. rapae crucivora in the short-wavelength region of the spectrum.

Key words: color vision; visual pigment; optical filter; insect; rhodopsin; compound eye

\section{Introduction}

The absorption spectrum of visual pigments is the primary determinant of the spectral sensitivity of photoreceptor cells. A set of photoreceptors with different spectral sensitivities enables color vision (Pichaud et al., 1999). The human trichromatic system is based on blue (B)-, green (G)-, and red-sensitive cone photoreceptors, each expressing a spectrally distinct visual pigment. The honeybee compound eye similarly has three photoreceptor types, however, expressing an ultraviolet (UV)-, B-, or G-absorbing visual pigment. The differences in the covered spectral ranges and the spectral tuning of the visual pigments used by the individual photoreceptors presumably depend on many evolutionary and ecological factors (Lythgoe, 1979; Briscoe and Chittka, 2001). Several animal species have diversified their color vision by developing multiple spectral photoreceptor classes, by duplicating visual pigment genes, as occurred with the human green and red cones, and/or installing pigments that act as spectral filters, like the carotenoids in the oil droplets of bird cones (Douglas and Marshall, 1999). An understanding of the evolutionary design principles underlying the spectral diversification of photoreceptors and their neural systems can be gained from combined stud-

Received April 8, 2005; revised May 11, 2005; accepted May 11, 2005

This work was supported by research grants from the Ministry of Education, Culture, Sports, Science, and Technology of Japan, Yokohama City, and the Mitsubishi Foundation to K.A.

Correspondence should be addressed to Dr. Kentaro Arikawa, Graduate School of Integrated Science, Yokohama City University, 22-2 Seto Kanazawa-ku, Yokohama 236-0027, Japan. E-mail: arikawa@yokohama-cu.ac.jp. DOI:10.1523/JNEUROSCI.1364-05.2005

Copyright $\odot 2005$ Society for Neuroscience $\quad$ 0270-6474/05/255935-08\$15.00/0 ies of the molecular biology of visual pigments, the spectral sensitivities of photoreceptors, and their potential in visual ecology. For such aims, insects, and particularly butterflies, offer excellent study cases (Bernard and Remington, 1991; Briscoe and Chittka, 2001; Stavenga, 2002; Arikawa, 2003).

According to molecular phylogeny, insect visual pigments can be divided into two main groups, the short- and long-wavelength visual pigments (Gärtner, 2000). The short-wavelength group can be further divided into UV and B visual pigments (Briscoe and Chittka, 2001). Although insect eyes generally seem to express a set of three visual pigments, several species have more than three classes of spectral photoreceptors: Drosophila (Salcedo et al., 1999); wasp, Tenthredo (Peitsch et al., 1992); and butterflies, Lycaena (Bernard and Remington, 1991) and Papilio (Arikawa, 2003). The sensitivity spectra occasionally deviate severely from the standard shape of visual pigment absorption spectra because of optical factors, of which spectrally selective filters are the most important (Arikawa et al., 1999a,b; Douglas and Marshall, 1999). For instance, in the eyes of the male small white butterfly, Pieris rapae crucivora, pale-red and deep-red pigment clusters that surround the rhabdoms act as long-pass filters, thus creating narrow-band red or deep-red spectral sensitivities (Qiu et al., 2002; Qiu and Arikawa, 2003b; Wakakuwa et al., 2004).

Here, we performed a comparative study of the female and male eyes of $P$. rapae crucivora, applying combined molecular biology, electrophysiology, and in vivo fluorescence microscopy. We found three mRNAs encoding opsins of novel short- 
wavelength visual pigments, with peak absorption in the UV, violet $(\mathrm{V})$, and $\mathrm{B}$ wavelength range. The expression patterns of the mRNAs are identical in both sexes, but the spectral sensitivity of the photoreceptors expressing the $\mathrm{V}$-absorbing visual pigment distinctly differs between the sexes, most likely because of a malespecific fluorescing pigment that acts as a selective $\mathrm{V}$ filter. $P$. rapae crucivora is the first animal species expressing three shortwavelength visual pigments, and the set of associated spectral photoreceptors is sex specific.

\section{Materials and Methods}

Materials. We used spring-form males and females of $P$. rapae crucivora. The butterflies were taken from a laboratory stock culture derived from eggs laid by females caught in the field around the campus of Yokohama City University. The hatched larvae were reared on fresh kale leaves at $19^{\circ} \mathrm{C}$ under a light regimen of $8 / 16 \mathrm{~h}$ light/dark. The pupae were stored at $4^{\circ} \mathrm{C}$ for at least 3 months and allowed to emerge at $25^{\circ} \mathrm{C}$ before experimentation. The adults were used within $4 \mathrm{~d}$ after emergence.

Anatomy. For studying the ommatidial heterogeneity, we fixed isolated eyes in $4 \%$ paraformaldehyde in $0.1 \mathrm{~m}$ sodium cacodylate buffer, $\mathrm{pH}$ 7.4, at room temperature for $30 \mathrm{~min}$ and embedded them in Spurr's resin. Unstained transverse sections of $10 \mu \mathrm{m}$ thickness were observed and photographed with regular transmission microscopy. Ommatidial autofluorescence was also observed in intact eyes using a fluorescence microscope (BX-60; Olympus, Tokyo, Japan) under both UV and B-V excitation (using the U-MNU and U-MNBV mirror unit, respectively; Olympus).

Electrophysiology. For electrophysiology, a butterfly was mounted on a plastic stage set in a Faraday cage. A silver wire inserted in the stump of an antenna served as the indifferent electrode. To insert a glass micropipette into the eye, a hole covering $\sim 10-20$ facets was made in the dorsal region of the cornea with a razor blade. The eye was then positioned at the center of a Cardan arm perimeter device. The dorsoventral axis of the compound eye was adjusted to a vertical orientation.

Monochromatic stimuli were provided by a $500 \mathrm{~W}$ xenon arc lamp through a series of narrow-band interference filters. The light was focused on the tip of an optical fiber that was attached to the perimeter device, where it provided a point source of light $\left(0.6^{\circ}\right.$ in diameter $)$. The quantum flux of each monochromatic stimulus was measured by a radiometer (model 470D; Sanso, Tokyo, Japan), and the maximum quantum flux of each monochromatic stimulus at the corneal surface was adjusted to $5.0 \times 10^{11}$ photons per $\mathrm{cm}^{2} / \mathrm{s}$.

A glass microelectrode filled with a fluorescing dye, Alexa Fluor 568 (maximum excitation, $576 \mathrm{~nm}$; maximum emission, $599 \mathrm{~nm}$ ), $1 \%$ in 50 mM potassium phosphate, $\mathrm{pH} 7$, the resistance of which was $\sim 100 \mathrm{M} \Omega$, was inserted into the eye through the hole made in the cornea. After impalement of the electrode into a photoreceptor, the optical axis of the photoreceptor was located by moving the tip of the optical fiber to maximize the response. We used flashes of $30 \mathrm{~ms}$ duration. First, the spectral sensitivity of the photoreceptor was determined by stimulating the cell with a series of monochromatic flashes. The stimulus intensity-response function was measured at the peak wavelength of the given photoreceptor over an intensity range of $5 \log$ units. The photoreceptor was subjected to additional analysis only when the maximal response amplitude was $>30 \mathrm{mV}$.

After recording, photoreceptors were marked by injecting Alexa Fluor 568 through the recording electrode by applying a $4 \mathrm{nA}$ hyperpolarizing current for $\sim 4 \mathrm{~min}$. The eyes were then processed for histology as described above. Unstained transverse sections of $10 \mu \mathrm{m}$ thickness were observed and photographed with regular transmission microscopy and fluorescence microscopy under green epi-illumination (with the U-MWIG mirror unit; Olympus) to determine the anatomical identity of the recorded photoreceptor and the type of ommatidium to which the cell belongs.

Molecular cloning. Retinal mRNA was extracted using the QuickPrep Micro mRNA purification kit (Amersham Biosciences, Uppsala, Sweden) from eyes rapidly frozen in liquid nitrogen. For amplifying the cDNAs of short-wavelength-absorbing opsins by reverse transcription
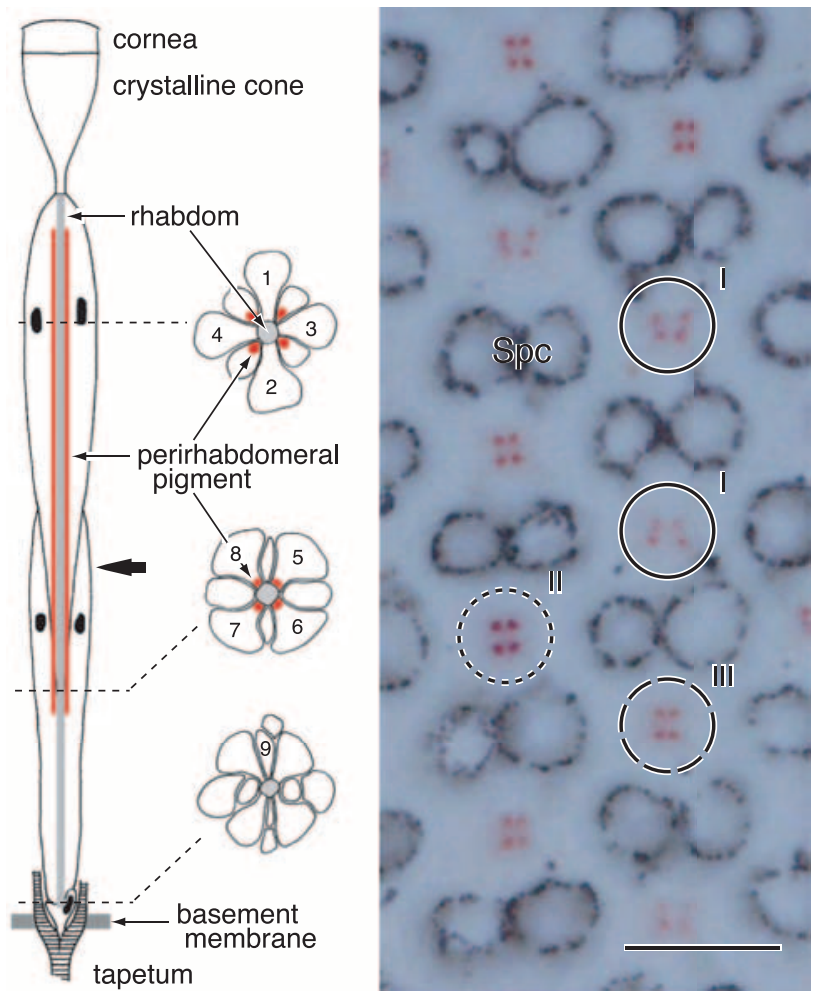

Figure 1. Anatomy of an ommatidium of the female $P$. rapae crucivora in the main frontoventral part of the compound eye. Left, The schematic diagram shows that an ommatidium contains nine photoreceptor cells R1-R9 (1-9), with four distal photoreceptors, R1-R4, four proximal photoreceptors, R5-R8, and one basal photoreceptor, R9. The three transverse views are from the distal, proximal, and basal levels (dotted lines) of the ommatidium. The rhabdomeres, the photoreceptor organelles that contain the visual pigment, are joined into the fused rhabdom. The rhabdom is surrounded by four clusters of pigment that exist in the distal extension of the soma of the proximal photoreceptors. The bold arrow indicates the level at which the micrograph on the right was taken. Right, Depending on the ommatidium, the cluster pattern is a trapezoid (I), square (II), or rectangle (III). The pigment is pale red in ommatidial types I and III and deep red in type II. Spc, Secondary pigment cells that optically isolate the ommatidia. Scale bar, $10 \mu \mathrm{m}$.

(RT)-PCR, we designed five degenerate primers based on amino acid sequences conserved in short-wavelength-absorbing opsins of the swallowtail butterflies Papilio xuthus (Kitamoto et al., 2000) and Papilio glaucus (Briscoe, 1998) and the hawkmoth Manduca sexta (Chase et al., 1997) (for regions, see Fig. 3). The PCR products were subcloned into pCRIITOPO vector and sequenced using the DYEnamic ET terminator cycle sequencing kit (Amersham Biosciences, Buckinghamshire, UK). To obtain the full-length cDNA, we performed $3^{\prime}$ - and $5^{\prime}$-rapid amplification of cDNA ends (RACE).

To compare the amino acid sequence deduced from the cloned cDNA with opsins of other insects identified so far, the sequences were aligned using an alignment program (Clustal $\mathrm{W}$ version 1.6), and a phylogenetic analysis was performed by the neighbor-joining method (Phylip version 3.572), having octopus opsin as an outgroup.

In situ hybridization. The compound eyes of male and female $P$. rapae crucivora were fixed in $4 \%$ paraformaldehyde in $0.1 \mathrm{M}$ sodium phosphate buffer, $\mathrm{pH} 7.2$, for $0.5-2 \mathrm{~h}$ at $25^{\circ} \mathrm{C}$. After dehydration with an ethanol series, we embedded the eyes in Paraplast (Oxford, St. Louis, MO). The Paraplast-embedded eyes were sectioned at $\sim 8 \mu \mathrm{m}$ thickness with a rotary microtome.

Probes for in situ hybridization were designed to hybridize to $\sim 400$ bases of the mRNA at the noncoding region downstream of the $\mathrm{C}$ terminus. The corresponding cDNA region was first subcloned into pGEM$3 \mathrm{zf}(+)$ vector, and then digoxigenin (DIG)-labeled cRNA was generated using the DIG-RNA labeling kit (Roche, Tokyo, Japan).

For labeling, sections were first deparaffinized and treated with hy- 

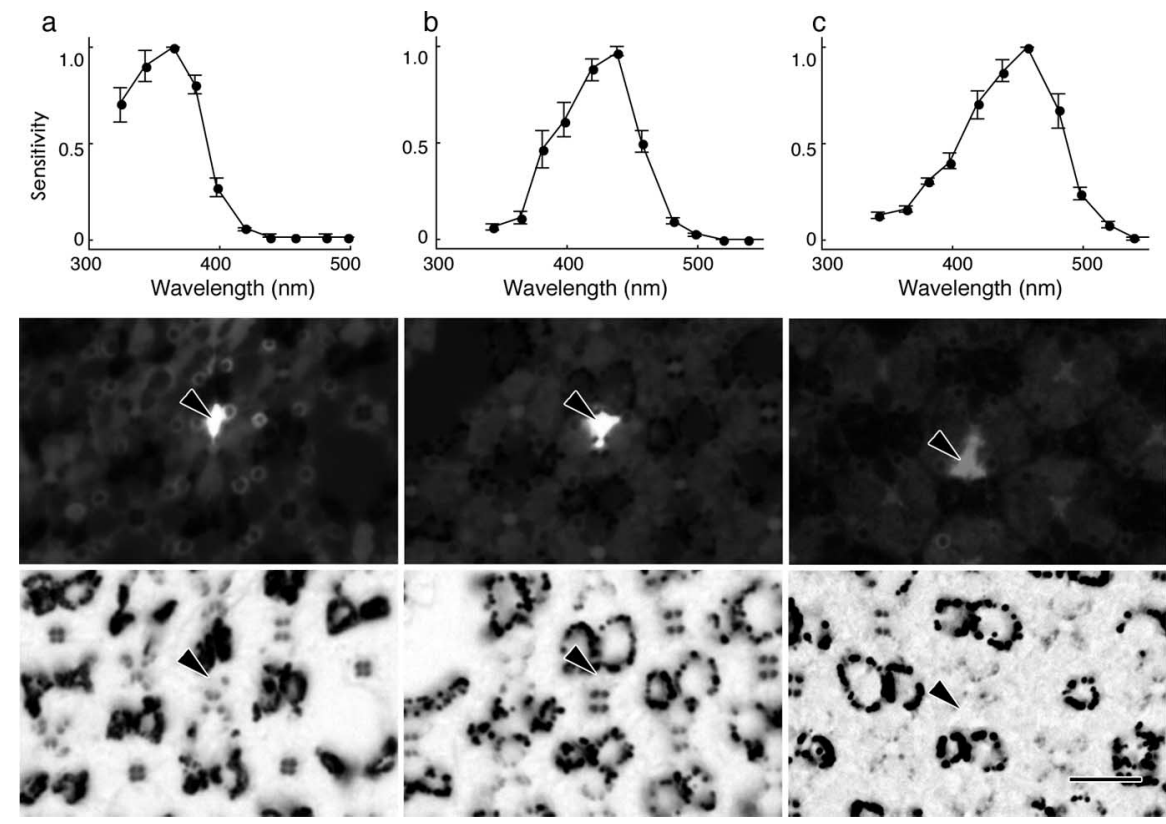

Figure 2. Spectral sensitivity of short-wavelength photoreceptors in female $P$. rapae crucivora and their localization (arrowheads). $\boldsymbol{a}$, A UV photoreceptor, peaking at $360 \mathrm{~nm}$ (top), identified as the R1 (middle) of a type I ommatidium (bottom). $\boldsymbol{b}, \mathrm{A} \mathrm{V}$ photoreceptor, peaking at $420 \mathrm{~nm}$ (top), identified as the R1 (middle) of a type II ommatidium (bottom). c, A B photoreceptor, peaking at $460 \mathrm{~nm}$ (top), identified as the R2 (middle) of a type I ommatidium (bottom). The spectral sensitivity curves are the means ( \pm SE) of six $(\boldsymbol{a})$, four $(\boldsymbol{b})$, and four $(\boldsymbol{c})$ consecutive measurements from single photoreceptors. Scale bar, $10 \mu \mathrm{m}$.

surrounded by clusters of red pigment in all ommatidia, except in a restricted dorsal eye area. The clusters exist in a distal extension of the soma of the proximal photoreceptors (Fig. 1, diagram). The pigment clusters vary both in shape and color, and, accordingly, three types of ommatidia can be distinguished. The shape of the pigment clusters in ommatidial types I, II, and III is trapezoidal, square, and rectangular, respectively, whereas the pigment color in both types I and III is pale red and that in type II is deep red (Fig. 1). The three types of ommatidia appeared to be arranged randomly in the main frontoventral eye region, in principle identical to that in the male small white butterfly (Qiu et al., 2002). We studied sections collected from 10 female individuals and found no obvious individual differences in the basic anatomy.

\section{Spectral sensitivity of \\ female photoreceptors}

We measured the spectral sensitivity of the photoreceptors of female $P$. rapae crucivora by intracellular recordings and identified the ommatidial type by dye injection. The spectral sensitivities of the

bridization solution (300 mм NaCl, 2.5 mм EDTA, 200 mм Tris- $\mathrm{HCl}, \mathrm{pH}$ $8.0,50 \%$ formamide, $10 \%$ dextran sulfate, $1 \mathrm{mg} / \mathrm{ml}$ yeast tRNA, and $1 \times$ Denhardt's medium) containing $0.5 \mu \mathrm{m} / \mathrm{ml}$ of the cRNA probe at $45^{\circ} \mathrm{C}$ overnight. After a brief rinse, the sections were incubated in $50 \%$ formamide in $2 \times \mathrm{SSC}$ at $55^{\circ} \mathrm{C}$ for $2 \mathrm{~h}$ and treated with RNase $(10 \mu \mathrm{m} / \mathrm{ml})$ at $37^{\circ} \mathrm{C}$ for $1 \mathrm{~h}$. The probes were further visualized by anti-DIG immunocytochemistry.

Modeling the absorbance spectrum of the V pigment filter. The in situ hybridization studies of the female $\mathrm{V}$ photoreceptors lead to the conclusion that these photoreceptors express the opsin PrV. The experimentally determined spectral sensitivity of the $\mathrm{V}$ receptor, $S_{\mathrm{V}}(\lambda)$, was assumed to be approximately equal to the absorption spectrum of the visual pigment. The latter spectrum can be derived from visual pigment templates (Stavenga et al., 1993; Govardovskii et al., 2000), which use the absorption peak wavelength, $\lambda_{\max }$, as the only parameter, yielding the absorption spectrum $R_{\lambda \max }(\lambda)$. Because the double-peaked blue (dB) photoreceptors also express PrV, a spectral filter presumably affected its spectral sensitivity. When $T(\lambda)$ is the transmittance of the pigment filter, the resulting spectral sensitivity is $S_{\mathrm{dB}}(\lambda)=T(\lambda) R_{\lambda \max }(\lambda)$. The absorbance spectrum of the filter pigment, $A_{\mathrm{fp}}(\lambda)$, equals the negative common logarithm of the transmittance, or the following (see Fig. 8):

$$
A_{\mathrm{fp}}=-\log _{10}(T)=\log _{10}\left(R_{\lambda \max }\right)-\log _{10}\left(S_{\mathrm{dB}}\right) .
$$

\section{Results}

\section{Ommatidial heterogeneity in female $P$. rapae crucivora}

Combined light- and electron-microscopic anatomy showed that the ommatidia in the compound eyes of the female small white butterfly, $P$. rapae crucivora, are tiered. In the distal tier, the rhabdomeres of four photoreceptors, R1-R4, together form the fused rhabdom, and in the proximal tier, this is done by the rhabdomeres of the photoreceptors R5-R8. The basal photoreceptor R9 contributes a very short basal part of the rhabdom. At the basal end, the rhabdom is abutted by the so-called tapetum, a tracheole that creates a multilayered stack that acts as an interference reflector (Ribi, 1979; Qiu et al., 2002). Transmission light microscopy of unstained sections reveals that the rhabdoms are photoreceptors R1 and R2 of the female small white butterfly peaked either in the UV, V, or B wavelength range, with peak wavelengths at $\sim 350,420$, and $460 \mathrm{~nm}$, respectively (Fig. 2 ). The $\mathrm{R} 1$ and R2 photoreceptors recorded in type I ommatidia were either UV or B photoreceptors, those in type II ommatidia were always only $\mathrm{V}$ photoreceptors, and those in type III ommatidia were UV photoreceptors (Table 1).

All recorded R3 and R4 photoreceptors in all three types of ommatidia had the same green spectral sensitivity, with peak wavelength at $\sim 560 \mathrm{~nm}$. The R5-R8 proximal photoreceptors were either red sensitive or deep-red sensitive, with peak wavelengths at $\sim 620 \mathrm{~nm}$ (LW620) and $\sim 640 \mathrm{~nm}$ (LW640), respectively. The LW620 photoreceptors were found exclusively in ommatidial types I and III, and the recorded LW640 were always localized in type II ommatidia. Data are not shown here, because the spectral sensitivities of the R3-R8 photoreceptors of the female appeared to be virtually indistinguishable from the corresponding photoreceptors in the male (Qiu and Arikawa, 2003a,b).

Comparing these results with the male, it appeared that ommatidial types I and III of both the female and male were organized identically. The type II ommatidia distinctly differed, however, because the type II R1 and R2 photoreceptors of the male had a $\mathrm{dB}$ spectral sensitivity. They were therefore termed $\mathrm{dB}$ photoreceptors (Qiu and Arikawa, 2003a).

\section{Three opsins of short-wavelength visual pigments}

The different spectral sensitivities of the R1 and R2 photoreceptors in type II ommatidia of female and male $P$. rapae crucivora raised the possibility that these photoreceptors use different visual pigments. We therefore analyzed the visual pigments of the short-wavelength photoreceptors by molecular biological experiments. We designed four forward primers (UV1F, UV2F, B1F, $\mathrm{B} 2 \mathrm{~F}$ ) and a reverse primer (R) and performed RT-PCR using 
Table 1. Short-wavelength photoreceptors of $P$. rapae crucivora and their visual pigments in the three types of ommatidia

\begin{tabular}{lllllllll}
\hline Ommatidia & Type I & \multicolumn{3}{l}{ Type II } & & & \multicolumn{2}{l}{ Type III } \\
\hline Pigmentation & Trapezoid & \multicolumn{2}{l}{ Square } & & & \multicolumn{2}{l}{ Rectangular } \\
Sex & Male/female & Male & & Female & & \multicolumn{2}{l}{ Male/female } \\
Fluorescence & No & & Yes & & No & & No & \\
\hline Photoreceptor & S $(\lambda)$ & Opsin & $S(\lambda)$ & Opsin & $S(\lambda)$ & Opsin & $S(\lambda)$ & Opsin \\
\hline R1 & UV & PrUV & dB & PrV & V & PrV & UV & PrUV \\
R2 & B & PrB & dB & PrV & V & PrV & UV & PrUV \\
\hline
\end{tabular}

mRNA extracted from the retinal tissue of a mixed population of male and female $P$. rapae crucivora. Through the RT-PCR, we eventually identified three novel cDNAs encoding shortwavelength visual pigment opsins (Fig. 3).

We analyzed 17 subclones obtained from the RT-PCR using the primer pair UV1F/R. Thirteen of them were fragments amplified from one mRNA (sq 1). The remaining four contained two different ones (sq 2 and 3). The primer pair UV2F/R yielded 12 subclones of sq 1 . Two primer pairs, $\mathrm{B} 1 \mathrm{~F} / \mathrm{R}$ and $\mathrm{B} 2 \mathrm{~F} / \mathrm{R}$, gave six and four subclones of sq 2, respectively. The B1F/R pair gave six clones of sq 3 as well, which were also used to identify this particular sequence by $3^{\prime}$ - and $5^{\prime}$-RACE, as we performed for other sequences. Based on the RACE results, we obtained full-length cDNAs of all of these three sequences by PCR using specific primers. Among the cDNA clones we analyzed, we found 6, 10, and 9 nucleotide substitutions in sq 1,2 , and 3, respectively, none of which affected the amino acid sequences deduced from the nucleotide sequences (Fig. 3).

A phylogenetic analysis of the amino acid sequences deduced for the three visual pigments indicated that they indeed belonged to the cluster of short-wavelength visual pigments (Fig. 4). Note that these opsins have a lysine at site 111 (PrUV), a glutamate at site $106(\mathrm{PrV})$, or a glutamate at site 109 (PrB), which have been shown to confer UV-absorbing (lysine) and B-absorbing (glutamate) spectra (Salcedo et al., 2003).

We localized the mRNAs of the three novel opsins in the retinal tissue by histological in situ hybridization (Fig. 5). In situ hybridization on transverse sections through the distal tier revealed that the R1 and R2 photoreceptors expressed only one of the three mRNAs, in all ommatidia of both females and males; no overlap was observed (Fig. $5 a-f$ ). All of the mRNAs were detected exclusively in the distal photoreceptors: the proximal and basal tiers of the ommatidia were free from labeling (Fig. $5 g-i$ ).

We correlated the labeling of photoreceptors with the three types of ommatidia by comparing three consecutive histological sections of female eyes, each labeled with a different probe for the three visual pigments. The outcome was that one probe labeled only one of the R1 and R2 photoreceptors in ommatidial type I, but it labeled both R1 and R2 in type III ommatidia (Fig. 5a). Because R1 and R2 in type III ommatidia were UV photoreceptors, we termed the visual pigment expressed by the corresponding DNA PrUV ( $P$. rapae ultraviolet-absorbing type). The second probe labeled exclusively, but simultaneously, the R1 and R2 of type II ommatidia (Fig. 5b). These photoreceptors were $\mathrm{V}$ photoreceptors, and therefore we termed their visual pigment $\operatorname{PrV}(P$. rapae violet-absorbing type). The third probe labeled the $\mathrm{R} 1$ or R2 of type I ommatidia complementing the photoreceptor labeled by the PrUV probe (Fig. $5 c$ ). The R1 and R2 of type I ommatidia were either UV or B photoreceptors, and therefore the third probe must have labeled the latter. We hence termed the visual pigment $\operatorname{PrB}(P$. rapae blue-absorbing type).

We performed the same series of in situ hybridization experiments on eyes of the male small white butterfly (Fig. $5 d-i$ ). The surprising result was that the labeling patterns were identical to those of the female, including the R1 and R2 photoreceptors of type II ommatidia, although the spectral sensitivities of the latter photoreceptors in the female and male strongly differed (Fig. 6b). The spectral sensitivities of the female UV, V, and B photoreceptors are reasonably well fitted by visual pigment templates peaking at 360, 425, and $453 \mathrm{~nm}$, respectively, and the PrUV, PrV, and PrB visual pigments were, accordingly, characterized as R360, R425, and R453 (Fig. $6 a-c$ ). The R1 and R2 photoreceptors in ommatidial type II of the male expressed R425, but the $\mathrm{dB}$ spectral sensitivity strongly deviated from the absorption spectrum of R425 (Fig. 6b). This induced the inevitable conjecture that the $\mathrm{dB}$ spectral sensitivity was attributable to a spectrally selective filter, suppressing the sensitivity of the male photoreceptors at the short-wavelength limb.

\section{Fluorescence microscopic observations}

Fluorescence microscopic observations of female versus male eyes, applying UV or V excitation, endorsed the hypothesis of spectral filtering (Fig. 7). Male eyes exhibited a quite prominent fluorescence, fully restricted to type II ommatidia (Qiu et al., 2002). The female eyes fluoresced only very faintly. A fluorescence comparable with that of the male Pieris was observed in the eyes of the Japanese yellow swallowtail, Papilio xuthus (both in the male and female), also exclusively seen in type II ommatidia. [Note that although Papilio and Pieris eyes have a similar anatomy, their spectral organization is quite different (Arikawa, 2003).] In Papilio, the fluorescent substance, most likely

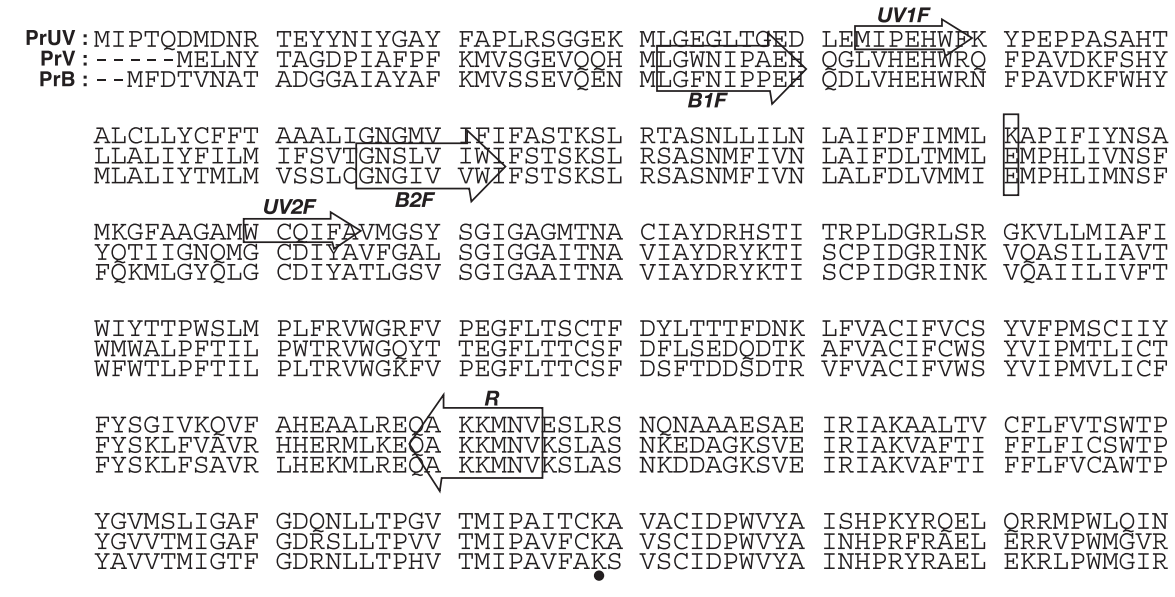

EPDDNTSTTS NTTTNSAAPA-- $\quad\left(\begin{array}{l}378 \\ 373\end{array}\right.$ amino acids $)$

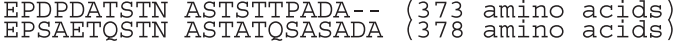

Figure 3. Deduced amino acid sequences of the three short-wavelength opsins: $\operatorname{PrUV}, \operatorname{PrV}$, and PrB. Nucleotide sequences are available in the DNA Data Bank of Japan (accession numbers: PrUV, AB208673; PrV, AB208674; PrB, AB208675). The boxed lysine and glutamate residues correspond to the sites responsible for short-wavelength-absorbing characteristics (Salcedo et al., 2003). The boxed arrows indicate regions on which forward (UV1F, UV2F, B1F, B2F) and reverse (R) primers were designed. The lysine residue indicated by the black dot is the site for chromophore attachment. 


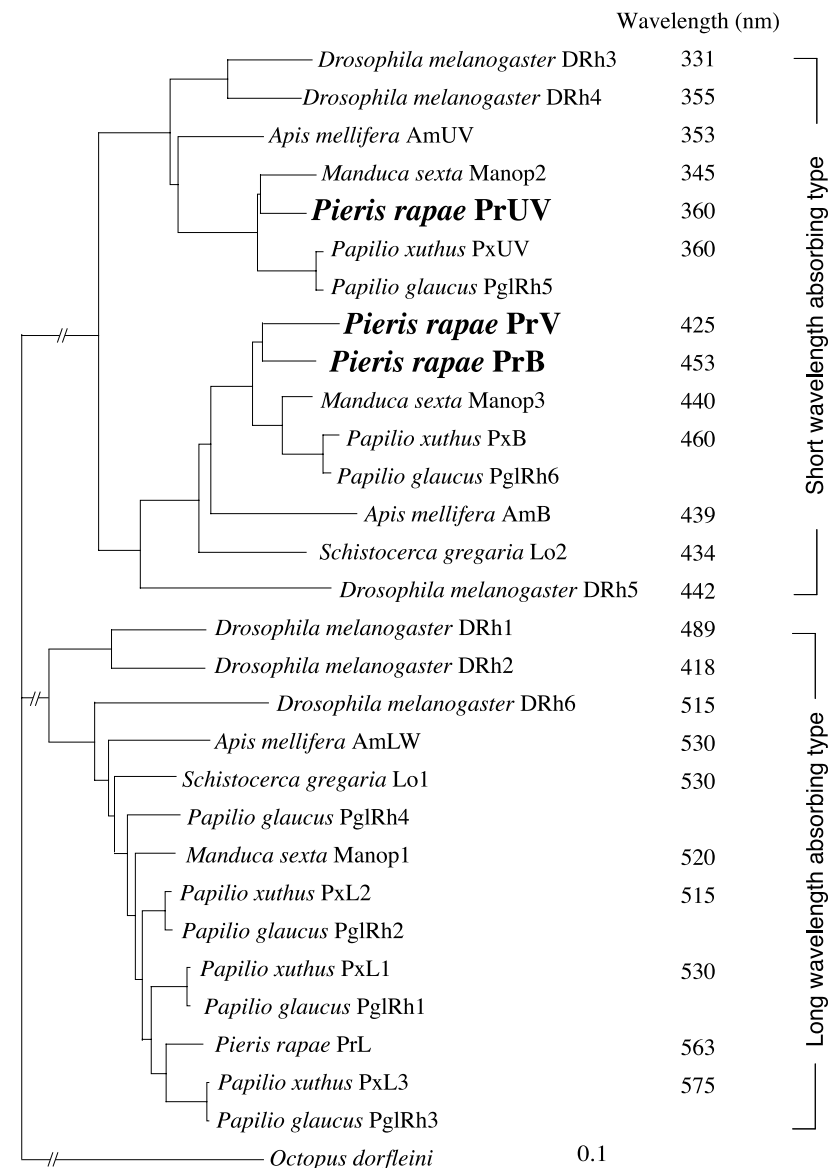

Figure 4. Phylogenetic relationship of insect visual pigment opsins. The data accession numbers are as follows: Apis mellifera AmB, AF004168; Apis mellifera AmLW, AF091732; Apis mellifera AmUV, AF004169; Drosophila melanogaster Rh1, K02315; Drosophila melanogaster Rh2, M12896; Drosophila melanogaster Rh3, M17718; Drosophila melanogaster Rh4, P08255; Drosophila melanogaster Rh5, U67905; Drosophila melanogaster Rh6, Y00043; M. sexta Manop1, L78080; M. sexta Manop2, L78081; M. sexta Manop3, AD001674; P. glaucus PglRh1, AF077189; P. glaucus PglRh2, AF077190; P. glaucus PgIRh3, AF067080; P. glaucus PglRh4, AF077193; P. glaucus PglRh5, AF077191; P. glaucus PglRh6, AF077192; P. xuthus PxB, AB028217; P. xuthus PxUV, AB028218; P. xuthus PxL1, AB007423; P. xuthus PxL2, AB007424; P. xuthus PxL3, AB007425; P. rapae PrL, AB177984; Schistocerca gregaria L01, X80071; Schistocerca gregaria L02, X80072. The peak wavelengths were taken from the studies by Salcedo et al. (1999) (Drosophila), Towner et al. (1997) (Schistocerca), Townson et al. (1998) (Apis), White et al. (2003) (Manduca), Engels et al. (2000) (Schistocerca), and Arikawa (2003) (Papilio).

3-hydroxyretinol, is localized within the rhabdom of type II ommatidia and acts there as a spectral filter for the UV visual pigment-containing $\mathrm{R} 1$ and $\mathrm{R} 2$ photoreceptors, thus causing a narrow-band, V-peaking spectral sensitivity (Arikawa et al., 1999b). We therefore hypothesized that the fluorescing substance in type II ommatidia of male Pieris eyes similarly acts as a spectral filter, modifying a $\mathrm{V}$ photoreceptor into a $\mathrm{dB}$ photoreceptor.

\section{Absorbance spectrum of the filter pigment in male type II ommatidia}

The absorbance spectrum of the filtering pigment was deduced from the spectral sensitivities as outlined in Materials and Methods. Figure $8 a$ presents the common logarithm of the normalized absorption spectrum of the $\mathrm{V}$ visual pigment, $\log _{10}\left[R_{425}(\lambda)\right]$, and of the spectral sensitivity of the $\mathrm{dB}$ photoreceptors, $\log _{10}\left[S_{\mathrm{dB}}(\lambda)\right]$. The latter spectrum is arbitrarily shifted along the ordinate so that the last two data points, at 460 and $480 \mathrm{~nm}$, approximately coincide with the first spectrum. The absorbance spectrum of the presumed pigment filter is then obtained from the difference between the two spectra of Figure $8 a$ (see Eq. 1 in Materials and Methods). The resulting spectrum (Fig. $8 b$ ) can be well approximated by a lognormal curve for absorption spectra of organic pigments (Metzler et al., 1985), yielding a maximal absorbance of 0.58 at $416 \mathrm{~nm}$ and a half-bandwidth of $60 \mathrm{~nm}$.

\section{Discussion}

\section{Sex-specific $\mathrm{V}$ and $\mathrm{dB}$ photoreceptors}

The eyes of female $P$. rapae crucivora consist of three types of ommatidia, as follows from the different patterning of the clusters of pale-red and deep-red pigments in the soma of the proximal photoreceptors R5-R8 (Fig. 1). The ommatidial heterogeneity is also seen in the specific expression of three types of shortwavelength visual pigments, termed $\operatorname{PrUV}, \operatorname{PrV}$, and $\operatorname{PrB}$, in the distal photoreceptors R1 and R2 (Table 1). The same ommatidial heterogeneity was found anatomically in the eyes of males (Arikawa, 2003; present results). However, females and males differ in the characteristics of ommatidial type II. We concluded that a $\mathrm{V}$-absorbing, $\mathrm{B}-\mathrm{V}$ fluorescing pigment in male type II ommatidia modifies a $\mathrm{V}$ photoreceptor into a $\mathrm{dB}$ photoreceptor (Figs. $6 b, 8$ ). Both females and males therefore use three shortwavelength photoreceptors; the female has UV, V, and B photoreceptors, and the male has $\mathrm{UV}, \mathrm{dB}$, and $\mathrm{B}$ photoreceptors (Fig. 6, Table 1). A previous electrophysiological study reported only $\mathrm{UV}, \mathrm{V}$, and $\mathrm{B}$ photoreceptors in the eye of $P$. rapae crucivora, which therefore must have concerned the female (Shimohigashi and Tominaga, 1991).

\section{Mechanism of spectral tuning by fluorescing pigment}

The eyes of both females and males of the Japanese yellow swallowtail butterfly, $P$. xuthus, contain a short-wavelengthabsorbing and distinctly fluorescing pigment in the type II ommatidia, which is involved in modifying the spectral sensitivity of a short-wavelength photoreceptor. By absorbing the far-UV, the pigment changes a UV photoreceptor, peaking at $360 \mathrm{~nm}$, into a narrow-band $\mathrm{V}$ photoreceptor peaking at $400 \mathrm{~nm}$. The absorbing pigment, most likely 3-hydroxyretinol, is concentrated in the distal portion of the rhabdom and therefore is well positioned as a spectral filter (Arikawa et al., 1999b; Kitamoto et al., 2000). The location of the filter pigment in the type II ommatidia of male $P$. rapae crucivora is unknown but most likely is also concentrated distally. For the filter acting on the R1 and R2 photoreceptors, an effective absorbance of $\sim 0.6$ follows from Figure $8 b$. The pigment filter will also act on the proximal photoreceptors, and its effective absorbance will be $>0.6$ [Wakakuwa et al. (2004), their Fig. 4c]

The chemical nature of the fluorescing substance in the type II ommatidia of male $P$. rapae crucivora is so far enigmatic. The eye of $P$. rapae crucivora contains a certain amount of 3-hydroxyretinol (Seki et al., 1987), which distinctly fluoresces under UV excitation but little under V excitation, opposed to that of the unidentified Pieris pigment; also, the absorbance spectra of the two pigments widely deviate [compare Fig. $8 b$ with Fig. 5 in the study by Arikawa et al. (1999b)]. Possible candidates for the unknown pigment are carotenoids or derivatives, which have been shown to exist in many visual systems, including the human eye, and which act as filter pigment in fly eyes (Kirschfeld and Franceschini, 1977). The derived absorbance spectrum of Figure $8 b$ has been tentatively approximated by a heuristic, smooth lognormal curve (Metzler et al., 1985). Carotenoid absorption spectra usually exhibit a characteristic fine structure, but its resolution requires much higher spectral sampling than that applied in the present electrophysiological experiments. 
Molecular design of butterfly eyes Colocalization of two short-wavelength and six or seven long-wavelength photoreceptors in a single ommatidium seems to be a general design principle of lepidopteran eyes (Arikawa, 2003; Briscoe et al., 2003; White et al., 2003; Sauman et al., 2005). The emerging picture is that the ommatidia can be generally divided into three types according to the specific set of two short-wavelength photoreceptors. Presently, the simplest case is that of Vanessa cardui and related nymphalids, in which two visual pigments are used by three sets of short-wavelength photoreceptors, R1 and R2, coexisting in single ommatidia, namely UV-B, UV-UV, and B-B (Briscoe et al., 2003). The other photoreceptors of the ommatidia all express the same $G$ visual pigment. They have the same G spectral sensitivity (Kinoshita et al., 1997), probably because spectral filter pigments are virtually absent. In these somewhat uncomplicated cases, the three spectral types of photoreceptors (UVB-G) will provide the basis of trichromatic vision.

The eyes of the papilionid P. xuthus also use two short-wavelength visual pigments [i.e., $\mathrm{UV}\left(\lambda_{\max }=360 \mathrm{~nm}\right)$ and $\mathrm{B}\left(\lambda_{\max }=\right.$ $450 \mathrm{~nm})$ ], but here the three sets of spectral sensitivities are UV-B, V-V, and B-B, existing in ommatidial types I, II, and III, respectively. Although the R1 and R2 photoreceptors of the type II ommatidia in Papilio express the UV visual pigment, they are narrow-band $\mathrm{V}$ photoreceptors, as a result of the short-wavelength filter pigment in the rhabdom (see above). R3 and R4, the other distal photoreceptors, use a G-absorbing visual pigment and thus are green sensitive. The proximal photoreceptors of $P$. xuthus have diversified, because those in type I and type III ommatidia express either an orange- or a G-absorbing, long-wavelength visual pig-

ment, with absorption peaks at 575 and $515 \mathrm{~nm}$, respectively. Furthermore, the visual pigments are filtered by red and yellow pigment clusters lining the rhabdoms, thus causing two longwavelength photoreceptor types, R and $\mathrm{G}$, with sensitivity peaks in the red and $\mathrm{G}$ wavelength range (Kitamoto et al., 1998; Arikawa et al., 1999a). The Papilio proximal photoreceptors in type II ommatidia are extraordinary: they contain both the orange- and G-absorbing visual pigment, which causes the abnormally broad spectral sensitivity stretching from 400 to $700 \mathrm{~nm}$ (Arikawa et al., 2003). Such coexpression of multiple visual pigments in single photoreceptors has not been detected in other lepidopteran insects other than Papilio species (Briscoe et al., 2003).

The eyes of $P$. rapae crucivora have a rather different set of spectral photoreceptors. In the female, the three pairs of R1 and $\mathrm{R} 2$ photoreceptors are also UV-B, V-V, B-B, but here the V photoreceptors rely on a genuine visual pigment, presumably created by gene duplication of the $\mathrm{B}$ visual pigment (Fig. 4). In the male,
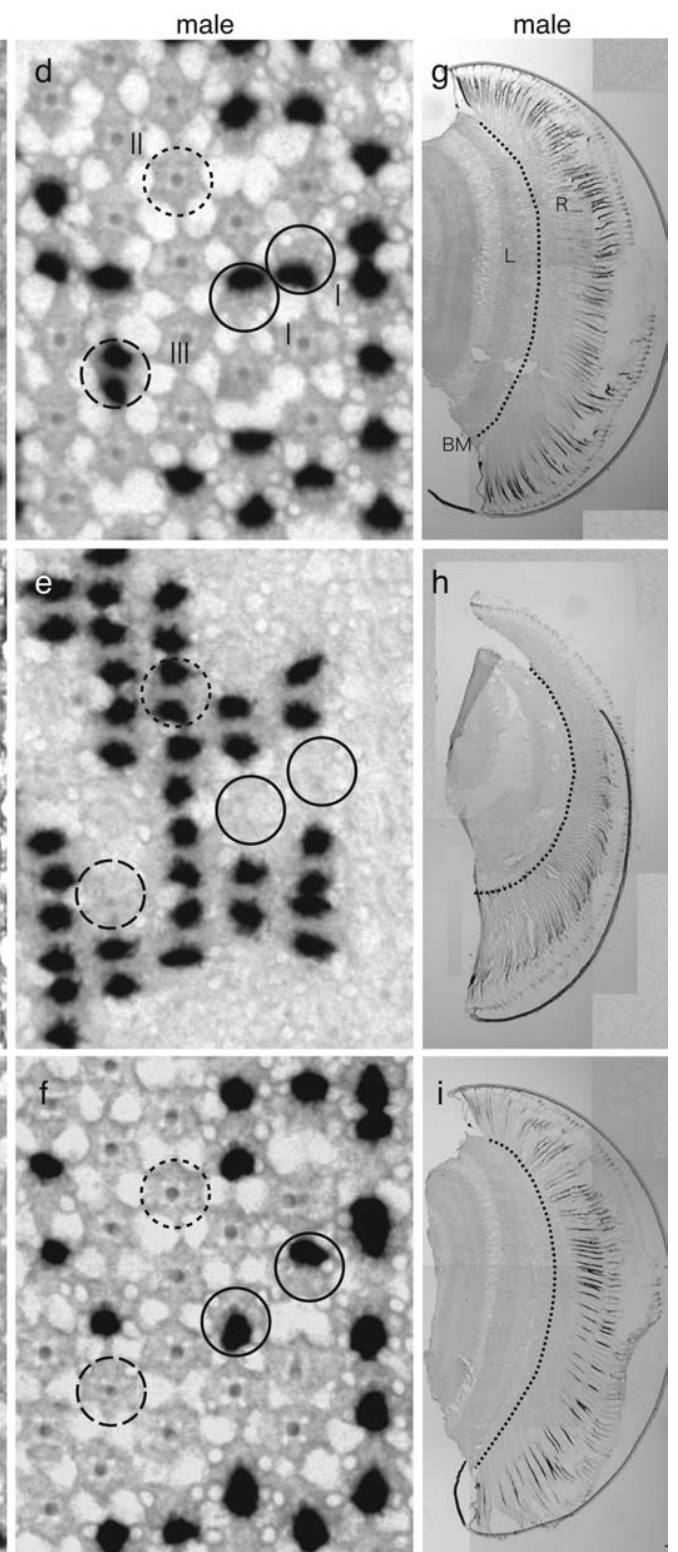

Figure 5. In situ hybridization of $\operatorname{PrUV}(\boldsymbol{a}, \boldsymbol{d}, \boldsymbol{g}), \operatorname{PrV}(\boldsymbol{b}, \boldsymbol{e}, \boldsymbol{h})$, and $\operatorname{PrB}(\boldsymbol{c}, \boldsymbol{f}, \boldsymbol{i})$ mRNAs in female $(\boldsymbol{a}-\boldsymbol{c})$ and male $(\boldsymbol{d}-\boldsymbol{i})$ P. rapae crucivora. I (filled circles), II (dotted circles), and III (dashed circles) indicate type I, II, and III ommatidia, respectively. BM, Basement membrane; $L$, lamina; $R$, retina.

the pairs are UV-B, $\mathrm{dB}-\mathrm{dB}, \mathrm{B}-\mathrm{B}$ (Table 1), in which filtering creates the $\mathrm{dB}$ photoreceptors from the PrV. The R3 and R4 photoreceptors of both males and females are $\mathrm{G}$ photoreceptors, because of a visual pigment peaking at $563 \mathrm{~nm}$, PrL. The proximal photoreceptors, R5-R8, of all ommatidia also express PrL, but depending on the pale-red or deep-red pigmentation around the rhabdom, the photoreceptors are red or deep-red photoreceptors, with very narrow sensitivity bands $\sim 620$ and $\sim 640 \mathrm{~nm}$ (Wakakuwa et al., 2004). The latter spectral types presumably serve in the discrimination of subtle color changes of green leaves in the long-wavelength range. Both females and males thus possess three long-wavelength photoreceptors (G-R-DR) in addition to the three short-wavelength photoreceptors (UV-V-B or UVdB-B), making a total of six different photoreceptor types in the main frontoventral part of the eyes. The spectral properties of the minor dorsal area remain to be explored.

Papilionidae and Pieridae are relatively closely related butter- 

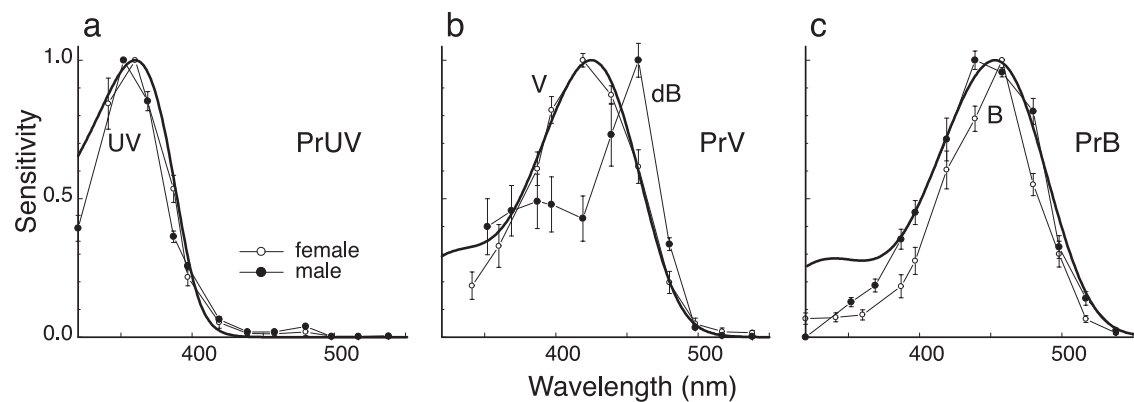

Figure 6. Average spectral sensitivity curves (error bars indicate SEs) of the three photoreceptor types, expressing the opsins $\operatorname{PrUV}(\boldsymbol{a}), \operatorname{PrV}(\boldsymbol{b})$, and $\operatorname{PrB}(\boldsymbol{c})$, recorded in female $(\bigcirc)$ and male $(\boldsymbol{O})$ P. rapae crucivora, together with visual pigment absorption spectra (Govardovskii et al., 2000) with absorption peaks at 360, 425, and $453 \mathrm{~nm}$, respectively (bold curves). The spectral sensitivities of UV $(\boldsymbol{a})$ and $\boldsymbol{B}(\boldsymbol{c})$ photoreceptors of males and females are similar and can be approximated reasonably well with a visual pigment spectrum. The same holds for the $V$ photoreceptors of the female, but not for the $\mathrm{dB}$ photoreceptors, of the male. All photoreceptors were identified histologically (as in Fig. 2). Six UV (a), three V (b), and five B (c) photoreceptors were measured in females. The spectral sensitivity curves for males were taken from the study by Qiu and Arikawa (2003a).
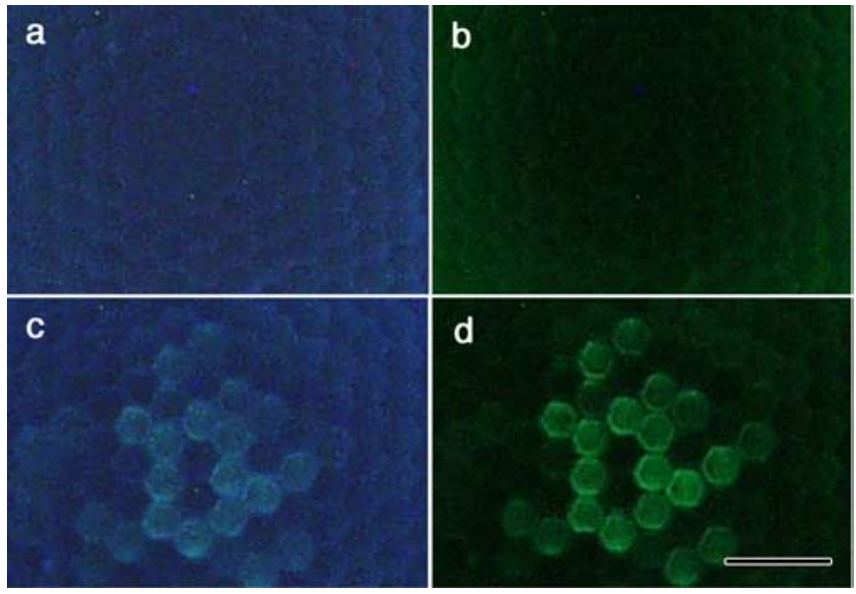

Figure 7. Fluorescence microscopic photographs of the eyes of female $(\boldsymbol{a}, \boldsymbol{b})$ and male $(\boldsymbol{c}, \boldsymbol{d})$ $P$. rapae crucivora, at the level of the corneal facet lenses, under UV $(\boldsymbol{a}, \boldsymbol{c})$ and violet $(\boldsymbol{b}, \boldsymbol{d})$ excitation. Whereas the fluorescence in female eyes is more or less homogeneous and faint, that of type II ommatidia in male eyes is distinct [for the relationship of the fluorescence and the ommatidial types, see Qiu et al. (2002)]. Scale bar, $50 \mu \mathrm{m}$.

a

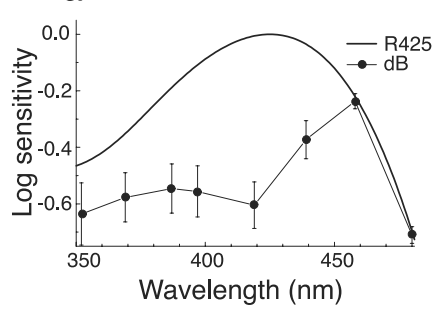

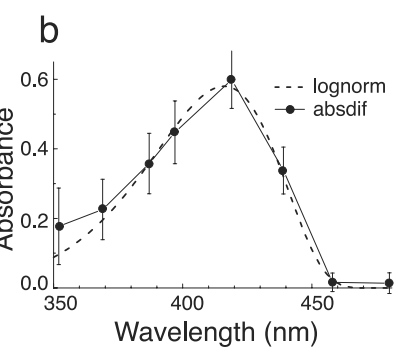

Figure 8. Derivation of the absorbance spectrum of the filter pigment in type ll ommatidia of male $P$. rapae crucivora. $\boldsymbol{a}$, The absorption spectrum of a visual pigment (rhodopsin) R425 and the spectral sensitivity of the $\mathrm{dB}$ photoreceptors (Fig. $6 b$ ). $\boldsymbol{b}$, The difference between the common logarithm of the spectra of $\boldsymbol{a}$ yields the absorbance spectrum (absdif) of the filter pigment. The dashed curve is calculated with the lognormal function of Metzler et al. (1985), with a peak absorbance of 0.58 , a peak wavelength of $416 \mathrm{~nm}$, a skewness of 1.7 , and a half-bandwidth of $60 \mathrm{~nm}$ (lognorm).

fly families, and both have developed three types of shortwavelength photoreceptors. However, the spectral characteristics and pigment combinations are quite different. The case of $P$. rapae crucivora is extraordinary, because it is the first animal species in which three different shortwavelength visual pigments with peak wavelengths within a range of $100 \mathrm{~nm}$ were identified. The modified spectral sensitivity of the $\mathrm{V}$ photoreceptors in the male is another novelty.

\section{Functional implications}

Why has the sexual dimorphism of the $P$. rapae crucivora eye come into existence? An attractive hypothesis is related to the striking difference in wing colors of females and males. Whereas the female wing reflectance is more or less constant throughout the visible wavelength range, including the UV, the male wing reflectance in the UV is minimal. Behavioral experiments have demonstrated that male $P$. rapae crucivora acutely discriminate the females by their high UV reflectance (Obara, 1970). Presumably, the fluorescing filter pigment of type II ommatidia of males has been recruited to improve spectral discrimination $\sim 400 \mathrm{~nm}$, where male wing reflectance abruptly changes. It is intriguing that the fluorescing pigment is absent in the eyes of males of the European subspecies P. rapae rapae (D. G. Stavenga, unpublished observation) and that the wing reflectances of the females and males are almost identical (Obara and Majerus, 2000; Stavenga et al., 2004). This suggests that the gamut of spectral photoreceptors, and the spectral discrimination system in which they participate, has developed in close conjunction with the spectral characteristics of their objects of interest.

\section{References}

Arikawa K (2003) Spectral organization of the eye of a butterfly Papilio. J Comp Physiol [A] 189:791-800.

Arikawa K, Scholten DGW, Kinoshita M, Stavenga DG (1999a) Tuning of photoreceptor spectral sensitivities by red and yellow pigments in the butterfly Papilio xuthus. Zool Sci 16:17-24.

Arikawa K, Mizuno S, Scholten DGW, Kinoshita M, Seki T, Kitamoto J, Stavenga DG (1999b) An ultraviolet absorbing pigment causes a narrow-band violet receptor and a single-peaked green receptor in the eye of the butterfly Papilio. Vision Res 39:1-8.

Arikawa K, Mizuno S, Kinoshita M, Stavenga DG (2003) Coexpression of two visual pigments in a photoreceptor causes an abnormally broad spectral sensitivity in the eye of a butterfly, Papilio xuthus. J Neurosci 23:4527-4532.

Bernard GD, Remington CL (1991) Color vision in Lycaena butterflies: spectral tuning of receptor arrays in relation to behavioral ecology. Proc Natl Acad Sci USA 88:2783-2787.

Briscoe AD (1998) Molecular diversity of visual pigments in the butterfly Papilio glaucus. Naturwissenschaften 85:33-35.

Briscoe AD, Chittka L (2001) The evolution of color vision in insects. Annu Rev Entomol 46:471-510.

Briscoe AD, Bernard GD, Szeto AS, Nagy LM, White RH (2003) Not all butterfly eyes are created equal: rhodopsin absorption spectra, molecular identification and localization of UV-, blue-, and green-sensitive rhodopsin encoding mRNA in the retina of Vanessa cardui. J Comp Neurol 458:334-349.

Chase MR, Bennett RR, White RH (1997) Three opsin-encoding cDNAs from the compound eye of Manduca sexta. J Exp Biol 200:2469-2478.

Douglas RH, Marshall NJ (1999) A review of vertebrate and invertebrate ocular filters. In: Adaptive mechanisms in the ecology of vision (Archer SN, Djamgoz MBA, Loew ER, Partridge JC, eds), pp 95-162. London: Kluwer Academic.

Engels A, Reichert H, Gehring WJ, Gärtner W (2000) Functional expression of a locust visual pigment in transgenic Drosophila melanogaster. Eur J Biochem 267:1917-1922.

Gärtner W (2000) Invertebrate visual pigments. In: Molecular mechanisms 
in visual transduction (Stavenga DG, DeGrip WJ, Pugh ENJ, eds), pp 297-388. Amsterdam: Elsevier.

Govardovskii VI, Fyhrquist N, Reuter T, Kuzmin DG, Donner K (2000) In search of the visual pigment template. Vis Neurosci 17:509-528.

Kinoshita M, Sato M, Arikawa K (1997) Spectral receptors of nymphalid butterflies. Naturwissenschaften 84:199-201.

Kirschfeld K, Franceschini N (1977) Photostable pigments within the membrane of photoreceptors and their possible role. Biophys Struct Mech 3:191-194.

Kitamoto J, Sakamoto K, Ozaki K, Mishina Y, Arikawa K (1998) Two visual pigments in a single photoreceptor cell: identification and histological localization of three mRNAs encoding visual pigment opsins in the retina of the butterfly Papilio xuthus. J Exp Biol 201:1255-1261.

Kitamoto J, Ozaki K, Arikawa K (2000) Ultraviolet and violet receptors express identical mRNA encoding an ultraviolet-absorbing opsin: identification and histological localization of two mRNAs encoding shortwavelength-absorbing opsins in the retina of the butterfly Papilio xuthus. J Exp Biol 203:2887-2894.

Lythgoe JN (1979) The ecology of vision. Oxford: Clarendon.

Metzler CM, Cahill AE, Petty S, Metzler DE, Lang L (1985) The widespread applicability of lognormal curves for the description of absorption spectra. Appl Spectrosc 39:333-339.

Obara Y (1970) Studies on the mating behavior of the white cabbage butterfly, Pieris rapae crucivora Boisduval III. Near-ultra-violet. Z Vergl Physiol 69:99-116.

Obara Y, Majerus MEN (2000) Initial mate recognition in the British cabbage butterfly, Pieris rapae rapae. Zool Sci 17:725-730.

Peitsch D, Fietz A, Hertel H, Desouza J, Ventura DF, Menzel R (1992) The spectral input systems of hymenopteran insects and their receptor-based colour vision. J Comp Physiol [A] 170:23-40.

Pichaud F, Briscoe A, Desplan C (1999) Evolution of color vision. Curr Opin Neurobiol 9:622-627.

Qiu X, Arikawa K (2003a) The photoreceptor localization confirms the spectral heterogeneity of ommatidia in the male small white butterfly, Pieris rapae crucivora. J Comp Physiol [A] 189:81-88.

Qiu X, Arikawa K (2003b) Polymorphism of red receptors: sensitivity spectra of proximal photoreceptors in the small white butterfly, Pieris rapae crucivora. J Exp Biol 206:2787-2793.

Qiu X, Vanhoutte KAJ, Stavenga DG, Arikawa K (2002) Ommatidial heter- ogeneity in the compound eye of the male small white butterfly, Pieris rapae crucivora. Cell Tissue Res 307:371-379.

Ribi WA (1979) Coloured screening pigments cause red eye glow hue in Pierid butterflies. J Comp Physiol [A] 132:1-9.

Salcedo E, Huber A, Henrich S, Chadwell LV, Chou WH, Paulsen R, Britt SG (1999) Blue- and green-absorbing visual pigments of Drosophila: ectopic expression and physiological characterization of the R8 photoreceptor cell-specific Rh5 and Rh6 rhodopsins. J Neurosci 19:10716-10726.

Salcedo E, Zheng L, Phistry M, Bagg EE, Britt SG (2003) Molecular basis for ultraviolet vision in invertebrates. J Neurosci 23:10873-10878.

Sauman I, Briscoe AD, Zhu H, Shi D, Froy O, Stalleicken J, Yuan Q, Casselman A, Reppert SM (2005) Connecting the navigational clock to sun compass input in monarch butterfly brain. Neuron 46:457-467.

Seki T, Fujishita S, Ito M, Matsuoka N, Tsukida K (1987) Retinoid composition in the compound eyes of insects. Exp Biol 47:95-103.

Shimohigashi M, Tominaga Y (1991) Identification of UV, green and red receptors, and their projection to lamina in the cabbage butterfly, Pieris rapae. Cell Tissue Res 263:49-59.

Stavenga DG (2002) Colour in the eyes of insects. J Comp Physiol [A] 188:337-348.

Stavenga DG, Smits RP, Hoenders BJ (1993) Simple exponential functions describing the absorbance bands of visual pigment spectra. Vision Res 33:1011-1017.

Stavenga DG, Stowe S, Siebke K, Zeil J, Arikawa K (2004) Butterfly wing colours: scale beads make white pierid wings brighter. Proc R Soc Lond B Biol Sci 271:1577-1584.

Towner P, Harris P, Wolstenholme AJ, Hill C, Worm K, Gärtner W (1997) Primary structure of locust opsins: a speculative model which may account for ultraviolet wavelength light detection. Vision Res 37:495-503.

Townson SM, Chang BS, Salcedo E, Chadwell LV, Pierce NE, Britt SG (1998) Honeybee blue- and ultraviolet-sensitive opsins: cloning, heterologous expression in Drosophila, and physiological characterization. J Neurosci 18:2412-2422.

Wakakuwa M, Stavenga DG, Kurasawa M, Arikawa K (2004) A unique visual pigment expressed in green, red and deep-red receptors in the eye of the small white butterfly, Pieris rapae crucivora. J Exp Biol 207:2803-2810.

White RH, Xu H, Munch T, Bennett RR, Grable EA (2003) The retina of Manduca sexta: rhodopsin-expression, the mosaic of green- blue- and UV-sensitive photoreceptors and regional specialization. J Exp Biol 206:3337-3348. 